\title{
LEFT SUPRACLAVICULAR LYMPHADENOPATHY AS THE ONLY CLINICAL PRESENTATION OF PROSTATE CANCER: A CASE REPORT
}

\author{
Mohanad Abusultan ${ }^{1}$, Hanzel $\mathrm{P}^{2}$, Durcansky $\mathrm{D}^{3}$, HaJtman $\mathrm{A}^{3}$. \\ ${ }^{1}$ Department of Otorhinolaryngology, Prievidza Hospital, Slovak Republic \\ ${ }^{2}$ Comenius University, Jessenius Faculty of Medicine and University Hospital in Martin, Clinic of \\ Otorhinolaryngology, Head and Neck Surgery, Martin, Slovak Republic \\ ${ }^{3}$ Department of Pathology, Prievidza Hospital, Slovak Republic
}

\begin{abstract}
Prostate cancer usually metastasis to the regional lymph nodes and can rarely metastases to nonregional supradiaphragmatic lymph nodes. Cervical lymph node metastasis of prostate cancer is extremely rare. However, it should be considered in the differential diagnosis of cervical lymphadenopathy in male patients with adenocarcinoma of unknown primary site. In this report we present a rare case of metastatic prostate adenocarcinoma with left supraclavicular lymphadenopathy as the only clinical presentation with no other evidence of metastasis to the regional lymph nodes or bone metastasis.
\end{abstract}

Key words: Prostate cancer, Supraclavicular lymphadenopathy, Metastasis

\section{INTRODUCTION}

Most of cancer metastasis to the cervical lymph nodes is from cancers of the mucosal surfaces of the upper aerodigestive tract. The second most common source of metastasis is nonmucosal tumors in the head and neck such as salivary glands, thyroid glands and skin [1]. Cancers originating from sites other than the head and neck can rarely metastasize to the cervical lymph nodes. However, neoplasms of the genitourinary tract make up a significant proportion of these cancers and should be considered in the differential diagnosis of neoplastic lesions of the head and neck [2]. Review of the literature suggests that in elderly male patients with neck masses, prostate carcinoma should be ruled out. Only few reports describe cervical metastasis as the first clinical presentation of prostate cancer, but in the setting of disseminated disease. We here present a very rare case of metastatic prostate adenocarcinoma with left supraclavicular lymphadenopathy as the only clinical presentation.

\section{CASE REPORT}

A 65-year-old male patient was admitted to the department of internal medicine for acute coronary syndrome (NSTEMI). Later patient had a Doppler ultrasound of the neck vessels with an incidental finding of multiple predominantly hypoechoic enlarged cervical lymph nodes, the largest node measuring $22 \times 15 \mathrm{~mm}$. After the stabilization of the patient he later

Address for correspondence:

MUDr. Pavel Hanzel, PhD, Clinic of Otorhinolaryngology and Head and Neck Surgery, Jessenius Faculty of Medicine, Comenius University and University Hospital Martin, Kollarova Str.2, 03601 Martin, Slovakia; Phone: +421-432633714; e-mail: pavelhanzel@gmail.com 
was referred to otorhinolaryngology. Patient has no other complaints, including fever, chills, weight loss, or difficulty swallowing or breathing. Physical examination revealed non-tender, semi-mobile lymph nodes in the left supraclavicular fossa. An ultrasound scan of the neck showed the presence of an enlarged lymph node on the left supraclavicular fossa with the size of 30x20mm, hypoechogenic, centrally markedly vascularized. Full head and neck clinical examination including flexible nasolaryngoscopy showed normal smooth mucosa of the oropharynx, hypopharynx, nasopharynx and larynx. Excisional biopsy of the left neck masses revealed poorly differentiated metastatic adenocarcinoma of undetermined origin. Immunohistochemical staining of the left supraclavicular lymph nodes was positive for PSA.

Patient was referred to the urologist were digital rectal exam revealed a hard prostate. Transrectal ultrasound guided needle biopsy of the prostate demonstrated adenocarcinoma (Gleason score $5+4=9$ ). Serum PSA $27,45 \mathrm{ng} / \mathrm{mL}$. A bone scan showed no bony metastases. $18 \mathrm{~F}$-choline PET/CT scan showed no signs of metastasis other than the left supraclavicular lymph nodes. Hormonal therapy was initiated. Eight months later, his left supraclavicular lymphadenopathy had regressed and serum PSA had decreased to 0,04 ng/mL. He remained without evidence of recurrent disease 12 months after treatment.

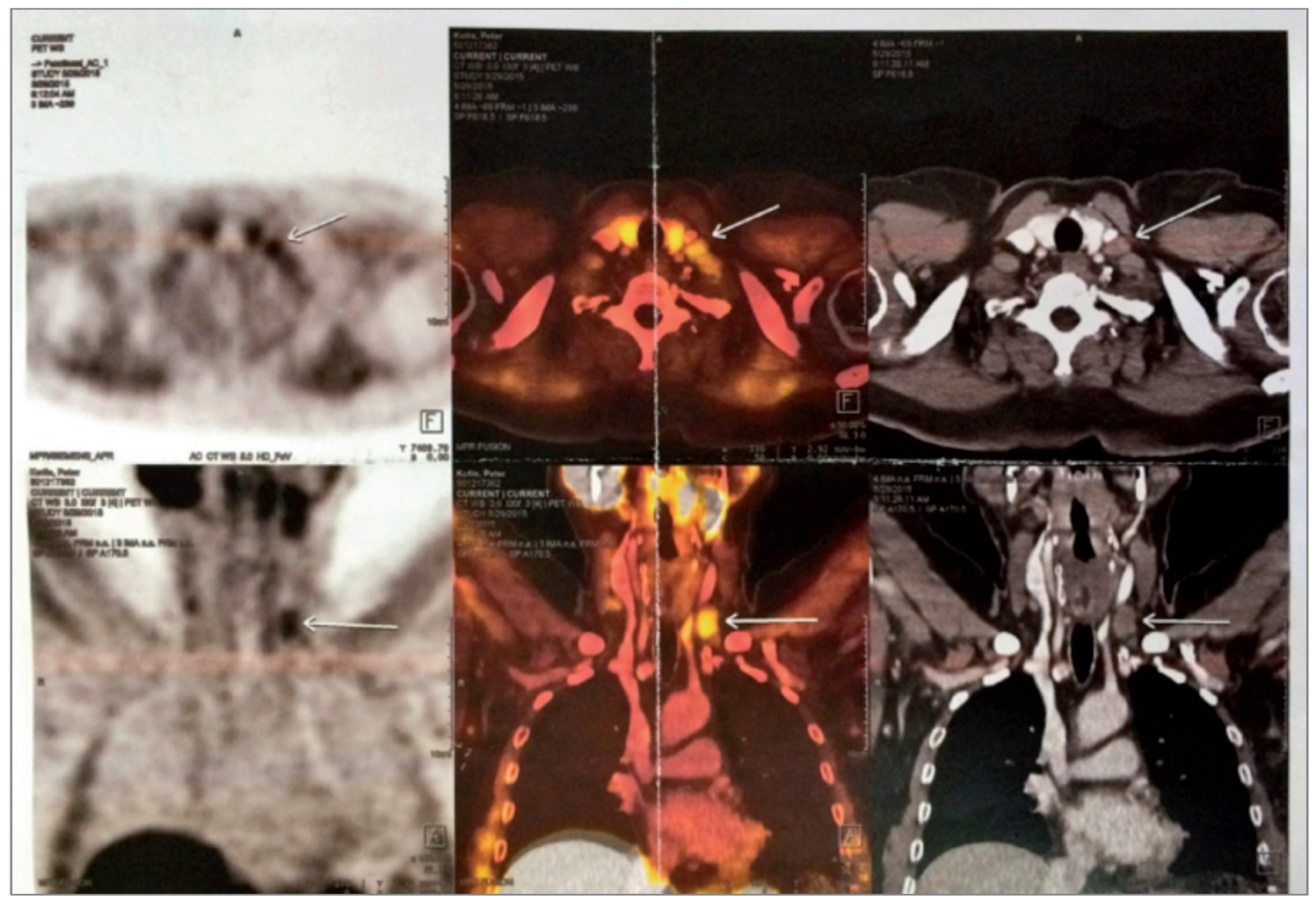

Fig. 1 18F-choline PET/CT scan shows enlarged supraclavicular lymph node in the left neck region 


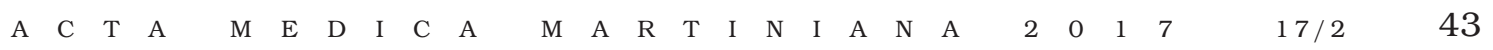

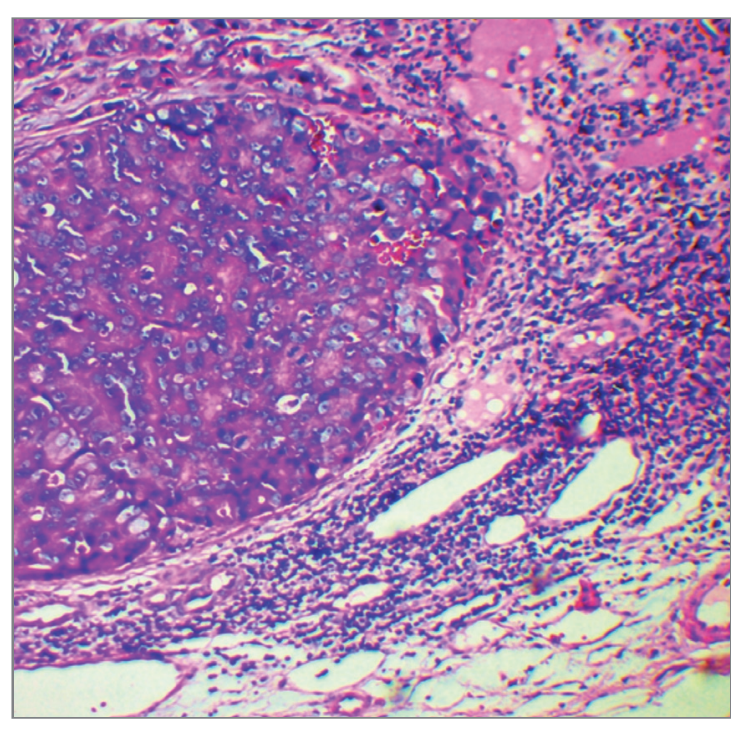

Fig. 2 Pathohistological examination shows solid growth pattern neoplasia, down residue lymph node (HE, obj. 10x).

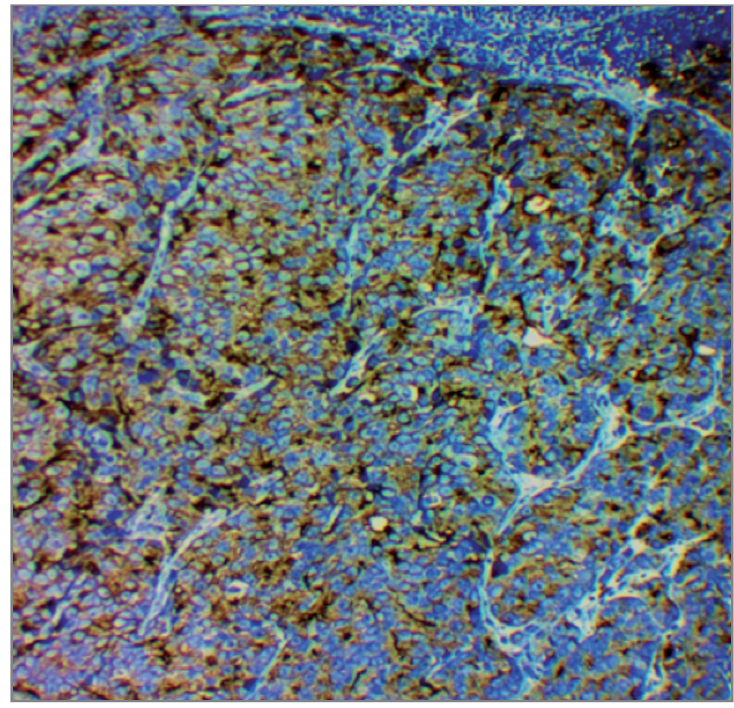

Fig. 3 Pathohistological examination shows Immunohistochemistry positivity evidence of prostate specific antigen (obj. 10x).

\section{DISCUSSION}

Prostate cancer is the most frequently diagnosed male cancer in Europe [3]. It represents the third most common cause of cancer-related death in Europe [3]. The spread of prostate carcinoma is known via three mechanisms: local extension, hematogenous spread, and lymphatic metastasis [4]. A common route of metastasis of prostate carcinoma is through lymphatic spread to the obturator, hypogastric, iliac, presacral and para-aortic nodes. The classical Virchow's node is the left supraclavicular node due to its location on the left side of the neck where most of the body's lymphatic drainage enters the venous circulation via the left subclavian vein. For this reason, many tumors of different origins have the tendency to metastasize to the left supraclavicular lymph nodes [6].

Despite the incidence and prevalence of prostate cancer and its tendency for metastasis, metastatic prostate carcinoma presenting as supraclavicular lymphadenopathy remains uncommon. However, this report in addition to other published reports, stress the need to consider metastatic prostate cancer in the differential diagnosis of a malignant lesions of the head and neck. The first autopsy study of prostate cancer metastasis was done by Mintz and Smith in 1934. Among the 100 autopsies on patients the prostate carcinoma, $60 \%$ of men had metastatic involvement of the lymph node and only one case of metastasis to the supraclavicular lymph node [5]. Flocks and Boatman did an extensive study of all urogenital malignancies and they found that 6 out of $1,500(0.4 \%)$ of patients with prostate cancer had evidence of cervical lymph node metastases on autopsy examination [6]. In a similar study of 1400 biopsies by Hematpour et al. only four cases of metastatic prostate cancer of the supraclavicular nodes were found [7].

The metastasis to the supraclavicular lymph nodes as the initial sign of prostate carcinoma has been reported rarely, usually on the left side [8,9]. Butler et al. described 19 patients with supraclavicular lymphadenopathy as the first presenting sign of prostate carcinoma, the left side lymph nodes were more commonly involved. [9] 
The metastasis of prostatic cancer to the head and neck can have a relatively good prognosis. Jones and Anthony presented 6 of 11 patients were alive and well after the diagnosis [10]. Wang et al reported 3 cases of prostate carcinoma presenting with left supraclavicular lymph node metastasis were hormonal therapy has been shown to be of benefit [11], which was the case in our patient who showed a regression of the supraclavicular lymphadenopathy and remained without evidence of recurrent disease after the hormonal therapy.

\section{CONCLUSION}

Although it's uncommon, prostate cancer should be considered in the otolaryngologist's differential diagnoses of supraclavicular lymphadenopathy in older males with adenocarcinoma of undetermined origin, even in the absence of lower urinary tract symptoms. Prostate cancer biopsy and Immunohistochemistry with PSA staining can confirm the diagnosis. Even in patients with an advanced stage of the disease, hormonal therapy is very effective.

\section{REFERENCES}

1. Copeland B, Clark JM, Sura A, Kilpatrick SE, Shockley W, Meredith S. Prostate carcinoma metastatic to the cervical lymph nodes: report of two cases and review of the literature. American journal of otolaryngology. 2001; 22(6):420-3.

2. Ogunyemi O, Rojas A, Hematpour K, Rogers D, Head C, Bennett C. Metastasis of genitourinary tumors to the head and neck region. European Archives of Oto-Rhino-Laryngology. 2010; 267(2):273-9.

3. Ferlay J, Steliarova-Foucher E, Lortet-Tieulent J, Rosso S, Coebergh JW, Comber H, Forman D, Bray F. Cancer incidence and mortality patterns in Europe: estimates for 40 countries in 2012. European journal of cancer. 2013; 49(6):1374-403.

4. Venable DD, Hastings D, Misra RP. Unusual metastatic patterns of prostate adenocarcinoma. The Journal of urology. 1983; 130(5):980-5.

5. MINTZ ER, SMITH GG. Autopsy findings in 100 cases of prostatic cancer. New England Journal of Medicine. 1934; $211(11): 479-87$.

6. Flocks RH, Boatman DL. Incidence of head and neck metastases from genito urinary neoplasms. The laryngoscope. 1973; 83(9):1527-39.

7. Hematpour K, Bennett CJ, Rogers D, Head CS. Supraclavicular lymph node: incidence of unsuspected metastatic prostate cancer. European Archives of Oto-Rhino-Laryngology and Head \& Neck. 2006; 263(9):872-4.

8. Chan G, Domes T. Supraclavicular lymphadenopathy as the initial presentation of metastatic prostate cancer: A case report and review of literature. Canadian Urological Association Journal. 2013; 7(56):E433.

9. Butler JJ, Howe CD, Johnson DE. Enlargement of the supraclavicular lymph nodes as the initial sign of prostatic carcinoma. Cancer. 1971; 27(5):1055-63.

10. Jones H, Anthony PP. Metastatic prostatic carcinoma presenting as left sided cervical lymphadenopathy: a series of 11 cases. Histopathology. 1992; 21(2):149-54.

11. Wang HJ, Chiang PH, Peng JP, Yu TJ. Presentation of prostate carcinoma with cervical lymphadenopathy: report of three cases. Chang Gung Med J. 2004; 27(11):840-4.

Received: June, 15, 2017

Accepted: August, 15, 2017 